\title{
FEQRP: a Fuzzy based Energy-efficient and QoS Routing Protocol over WSNs
}

\author{
Ahmed Hamood M. Al-Quh ${ }^{1}$, Prof. Imane Aly Saroit Ismail², \\ Dr. Amira Mohammed Kotb ${ }^{3}$ \\ I(Information Technology Department, Faculty of computers and information / Cairo University, Egypt) \\ 2 (information Technology Department, Faculty of computers and information / Cairo University, Egypt) \\ ${ }^{3}$ (information Technology Department, Faculty of computers and information / Cairo University, Egypt)
}

\begin{abstract}
In this paper, an energy efficient and QoS routing protocol; named Fuzzy based Energy-efficient and QoS Routing protocol (FEQRP) for wireless sensor networks; is proposed to extend the life time of the network. Most of the existing protocols don't take care about energy balancing. The energy manager protocols balance the energy consumption in the network to avoid network partitioning. The proposed protocol tries to find the best route with smallest distance between source and destination, reduce the energy consumption and balance it by fuzzy set approach. In FEQRP, both the node position and the energy level are used to route the packets from sources to destination. Each node knows the location and energy level of its neighbors.
\end{abstract}

Keywords: QoS, Routing protocol, Wireless Sensor Networks, Energy consumption

\section{Introduction}

Wireless sensor networks (WSNs) have gained worldwide attention in the recent years. It is enabled by recent advances in micro-electronic-mechanical systems (MEMS) and wireless communication technologies which has facilitated the development of smart sensors. Smart sensor nodes are low cost, low power and multifunctional devices that are deployed in a region of interest. It is equipped with one or more sensors, a processor, a memory, a power supply, an actuator and a radio. Battery is the main energy source in a sensor node. Secondary power supply that harvests power from the environment such as solar panels may be added to the node depending on the appropriateness of the environment where the sensor will be deployed. Depending on the application and the type of sensors used, actuators may be incorporated in the sensors [1].

Wireless sensor networks are used in many applications, such as natural disaster relief, military target tracking and surveillance, seismic sensing, hazardous environment exploration and biomedical health monitoring [2][3].

In recent years; the researchers developed quality of service $(\mathrm{QoS})$ routing protocols to reduce the energy consumption and prolong the lifetime of the WSNs. QoS requirements in the routing protocols is one of the most important challenges. QoS routing deals with the end-to-end delay, bandwidth, packet loss, packet reception rate and surplus energy metrics, and tries to find the path with the minimum resource consumption.

Providing an energy efficient and QoS on the routing protocol remains an open research issue. Most of the energy and QoS aware routing protocols in WSNs are designed to save the total energy consumption but don't focus on energy balancing. They try to reduce the energy consumption of the network as a whole, but it has poor idea about energy management. However the energy manager protocols balance the energy consumption in the WSN to avoid network partitioning. Therefore, a new QoS routing protocol over WSNs, named, Fuzzy based Energy-efficient and QoS Routing protocol over WSNs (FEQRP) is proposed, which considers energy saving as well as energy balancing by fuzzy set approach [4][5].

The rest of this paper is organized as follows: Section 2 presents the related work. Section 3 describes the proposed algorithm. Section 4 describes the details of the simulation model. Performance evaluation is presented in section 5. Section 6 concludes this paper.

\section{Related Work}

Energy and QoS routing in wireless sensor networks is a challenging problem because of the scarce resources of the sensor node. Thus, this problem has received a significant attention from the research community, where many proposals were made.

One of the early proposed routing protocols that provide QoS is the Sequential Assignment Routing (SAR) protocol [6]. SAR protocol is a multi-path routing protocol that makes routing decisions based on three factors: energy resources, QoS on each path and packet's priority level. Multiple paths are created by building a tree where the source node acts as its root. During paths construction, those nodes with low QoS and low 
residual energy are avoided. Employing multiple paths increases fault tolerance, but SAR protocol suffers from the overhead of maintaining routing tables and QoS metrics at each sensor node.

SPEED protocol is another QoS based routing protocol that provides soft real-time end-to-end guarantees paths [7]. Each sensor node maintains information about its neighbors and exploits geographic forwarding to find the paths. To ensure packet delivery within the required time limits, SPEED enables the application to compute the end-to-end delay by dividing the distance to the sink by the speed of the packet delivery before making any admission decision. Furthermore, SPEED can provide congestion avoidance when the network is congested. However, it does not take into account the energy of the forwarding nodes in order to balance the node energy utilization. Furthermore, the region it chooses for forwarding and the priority selection does not dynamically depend on the deadlines of the packets.

Greedy Perimeter Stateless Routing (GPSR) is the well-known and most commonly used positionbased routing protocol for WSNs [8]. GPSR works as follows: The source periodically uses a location service scheme to learn about the latest location information of the destination and includes it in the header of every data packet [9]. If the destination is not directly reachable, the source node forwaFrds the data packet to the neighbor node that lies closest to the destination. Such a greedy procedure of forwarding the data packets is also repeated at the intermediate nodes until the packet reaches to its destination. During greedy forwarding for GPSR, the available energy at the chosen neighbor node to forward the data packet is not considered.

Energy Aware Geographic Routing Protocol (EAGRP) is a geographic routing protocol for WSNs that guarantees QoS and at the same time minimizes energy consumption [10]. It takes into consideration both the node location and the energy consumption for making routing decisions. The EAGRP optimized the greedy forwarding phase of GPSR. It considered the energy available at the neighbor nodes of forwarding node before deciding the next hop node for transmitting the data packet.

In FEQRP protocol, we try to satisfy the QoS requirements with the minimum energy consumption. The proposed protocol tries to minimize the end to end delay, find the best route with the smallest distance between source and sink nodes, reduce the energy consumption and balance it through the WSN.

\section{The Proposed Fuzzy Based Energy-Efficient And Qos Routing Protocol Over Wsns (Feqrp)}

In this section the proposed FEQRP algorithm will be illustrated. This section is divided into 2 subsections. The first subsection will illustrate the fuzzy set, and the second subsection will describes the proposed algorithm.

\subsection{Fuzzy Set}

The theory of fuzzy sets was introduced by Prof. L. Zadeh in 1965 [5]. After the pioneering work of Prof. Zadeh, there has been a great effort to obtain fuzzy analogues of classical theories. Fuzzy set theory is a powerful tool for modeling uncertainty and for processing vague or subjective information in mathematical models. Their main directions of development have been diverse and its applications to the very varied real problems. The notion central to fuzzy systems is that truth values (in fuzzy logic) or membership values (in fuzzy sets) are indicated by a value in the range $[0,1]$, with "0" and "1" representing absolute Falseness and absolute Truth respectively [11].

In the proposed FEQRP algorithm a new scenario based on the fuzzy set technique is presented to balance the energy consumption in the network in order to avoid the network partitioning.

A is the fuzzy set of all neighbors' energy levels:

$$
\mathrm{A}=\left\{\mathrm{e}_{1}, \mathrm{e}_{2}, \ldots, \mathrm{e}_{\mathrm{n}}\right\}
$$

A has a membership function, $\mathrm{mA}(\mathrm{ei})$ which can be defined as below:

$$
\mathrm{mA}\left(\mathrm{e}_{\mathrm{i}}\right)=\lambda \mathrm{e}_{\mathrm{i}}, 1 \leq \mathrm{i} \leq \mathrm{n}
$$

Where $\lambda$ is a control parameter to limit the energy factor in $[0,1]$ interval, and ei is the energy level of (i $)_{\text {th }}$ neighbor.

The energy threshold used is obtained from the following formula:

$$
\alpha=\frac{\sum_{i=1}^{n} m A(e i)}{n} \text { then } A \alpha=\{e i \mid m A(e i) \geq \alpha\}
$$

Where $\alpha$ is energy threshold, and $A \alpha(\alpha-c u t)$ is used to remove the neighbors with unacceptable energy level.

\subsection{FEQRP Algorithm}

The FEQRP is divided into three stages. In the first stage, as the EAGRP algorithm, the location and the distance between the nodes are specified. In the second stage, balancing the energy consumption (proposed to the old algorithm) is calculated. The weight of the acceptable nodes (the residual energy plus the distance) is 
computed to choose the next hop in the last stage. The flow chart of the proposed FEQRP algorithm is shown in Fig. 1.

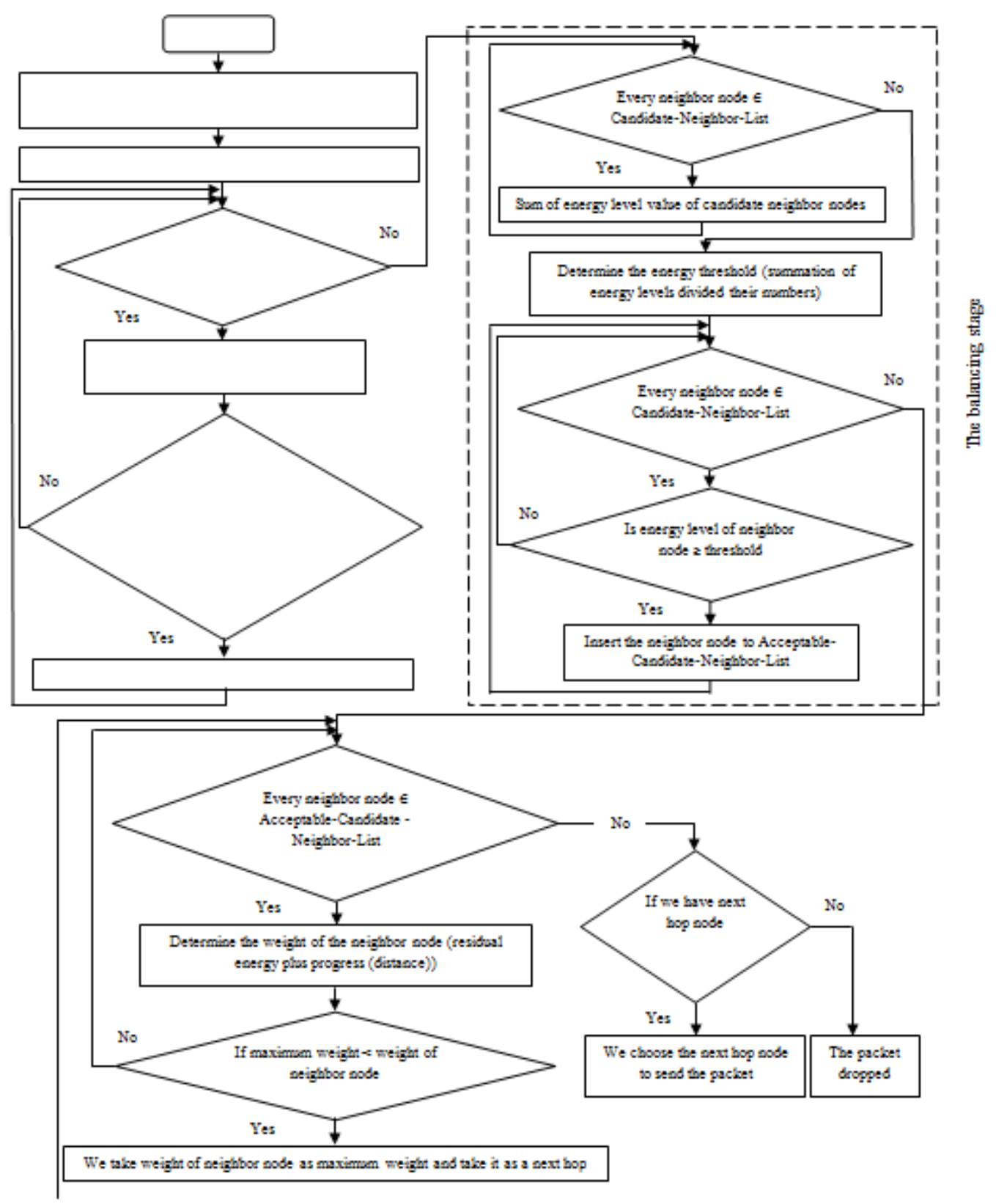

Fig. 1: Flow Chart for the proposed FEQRP algorithm

In the first stage, the FEQRP Algorithm first finds the location of all the nodes in the network. Then, the source node determines all its neighbors and put them in the neighbor list. The source node must have the location of the sink node to transmit the packet. Thus, the source node calculates the distance from itself to the sink node. Then, the source node calculates the distance between each one of its neighbors and the sink node. From the neighbor list, the source node sets the candidate set which contains the neighbor nodes that lie closer to the sink node than itself.

In the second stage, for the candidate set, the energy threshold is computed. It defined as:

$$
\text { Energy threshold }=\frac{\sum_{i=1}^{n} \text { (energy level for candidate node(i)] }}{n}
$$

Where $\mathrm{n}$ is the number of candidate neighbor nodes.

Then, the source node determines the acceptable set; the nodes that have energy level more than or equal to the energy threshold. Therefore, the acceptable candidate neighbor nodes are specified to choose the next hop. 
In the last stage, the weight of each acceptable candidate node is then computed. It is the sum of the residual energy of the acceptable candidate node plus its progress (the fraction of the distance covered between the forwarding node and the destination). The acceptable candidate node that has the maximum weight value is chosen by the source node as the next hop node to receive the data packet. These steps will continue till the packet reaches the sink node and all other packets will follow the same procedure.

\section{Simulation Model}

The OPNET is one of the most popular discrete event network simulation tools. OPNET provides a comprehensive development environment for modeling and performance evaluation of the communication networks and distributed systems. It was used to simulate the FEQRP and EAGRP algorithms to compare between their performances.

\subsection{Simulation Setup}

The nodes in the network are static. The irregular random topology for the sensor has been considered. Single destination (sink node) scenario is taken into account. In the simulation, all nodes generate data packets that are routed to the sink node.

The simulation system ran on two cases. The first case considers different network sizes (20, 40, 50, $65,75,85$, and 100 nodes) with fixed packet rate ( 2 packets/sec). The second case where different packet rates $(2,3,4,5,6$, and 7 packets/sec) and fixed number of nodes (65 nodes) are used. The simulation time for each network size was set to 500 seconds and repetitive simulations for each network size were performed to verify the reliability of the results. The network area was modeled on $300 \mathrm{x} 300$ meters.

All nodes in each scenario are considered as source nodes and generated data packets with bit rat of $11 \mathrm{Mb} / \mathrm{sec}$. The MAC layer uses the 802.11 wireless standards. The simulation parameters are summarized in Table 1.

Table 1: System Parameters Used in the Simulation Experiments.

\begin{tabular}{|c|c|}
\hline Simulation time & $500 \mathrm{sec}$ \\
\hline Simulation area & $300 \mathrm{~m} \times 300 \mathrm{~m}$ \\
\hline Number of nodes & $20,40,50,65,75,85$, and 100 \\
\hline Packet rates & $2,3,4,5,6$, and 7 packets/sec \\
\hline MAC type & IEEE802.11 \\
\hline Data rate & $11 \mathrm{Mb} / \mathrm{sec}$ \\
\hline Initial node energy & 1 joule \\
\hline
\end{tabular}

\subsection{Performance Metrics}

To evaluate the performance of the proposed FEQRP, five performance metrics will be calculated. These performance metrics are the average energy consumption, throughput, data dropped, average packet delivery ratio and average end-to-end delay.

\subsubsection{Average Energy Consumption (joule)}

It measures the average energy consumption per node through the simulation time. It is calculated as follows:

$$
\text { Average energy consumption }=\frac{\sum_{i=1}^{n} \text { energy consumed by each node) }}{n}
$$

Where $\mathrm{n}$ is the total number of nodes.

\subsubsection{Throughput (Kbits/sec)}

The total number of data packets delivered successfully over the simulation time; it is measured as the total number of delivered bits per second. It is calculated as follows:

$$
\text { Throughput }=\frac{\sum_{i=1}^{n}(\text { packets delivered }) \times \text { packet size }}{\text { simulation time }}
$$

\subsubsection{Data dropped (Kbits/sec)}

A data packet is dropped in two cases; the first case when a packet needs to be buffered and the buffer is full, the second case when time a packet has been buffered exceeded the limit. It is calculated as follows:

$$
\text { Data dropped } \left.=\left(\sum_{n} \text { (packets sent }\right)-\sum_{n}(\text { packets received })\right) \times \text { packet size }
$$




\subsubsection{Average packet delivery ratio}

It measures the ratio of the data packets received successfully by the sink to the data packets generated by the sources. It measured as follows:

$$
\text { Average packet delavery ratio }=\frac{\sum_{i=1}^{n}(\text { packets received })}{\sum_{i=1}^{n}(\text { packets received })} \times 100
$$

\subsubsection{Average end-to-end delay (sec)}

It measures the period of time taken to route the data packet from the source node to the sink node:

$$
\text { Average end }- \text { to }- \text { end delay }=\frac{\sum_{i=1}^{n}(\text { packets received time }- \text { packets sent time })}{\sum_{i=1}^{n}(\text { packets received })}
$$

\section{Performance Evaluation}

In this section the performance of the FEQRP algorithm will be evaluated and compared to that of the EAGRP algorithm. This section is divided into two subsections. The first subsection will illustrate the first case where variable network size is used with fixed packet rate. The second subsection will show the second case where variable packet rates are used with fixed network size.

\subsection{Case 1: Variable Network Size with Fixed Packet Rate}

Here, the five performance metrics will be discussed for both FEQRP and EAGRP algorithms. The network size varies between $(20,100)$ nodes while the packet rate is fixed to 2 packet/sec.

\subsubsection{Average Energy Consumption (joule)}

Fig. 2 shows the energy consumption of the proposed FEQRP and EAGRP algorithms. It is clear from the Fig. that the proposed FEQRP algorithm consumes less energy compared to that of the EAGRP algorithm. This is due to the selection of the fuzzy set technique at the neighbor node to choose the nodes with limited energy level to relay the data from the source node to the sink node. In the EAGRP, the source node chooses the next node from a candidate neighbor list with the maximum weight of progress (i.e. distance cover) plus the available energy. The proposed FEQRP determines the average level of energy to all nodes in the candidate neighbor list, and then remove the nodes with energy level more than the energy threshold. Therefore the FEQRP fairly balance and save the energy consumption in the network. This helps to significantly extend the lifetime of the network in heavy traffic scenarios.

\subsubsection{Throughput (Kbits/sec)}

Fig. 3 shows the throughput of the proposed FEQRP, and EAGRP algorithms. The throughput depends on the number of nodes, the number of packets transmitted at each node per second (packet rate), and the packet length. Increasing the number of nodes will lead to an increase in the throughput for both algorithms. From the Fig. it is clear that the FEQRP achieves higher throughput than that of the EAGRP. This is due to the fact that the EAGRP lacks to the energy balancing. So some nodes dies dramatically faster than others, therefore it suffers from network partitioning which leads to data loss.

\subsubsection{Data dropped (Kbits/sec)}

The data dropped is illustrated in Fig. 4 for both FEQRP and EAGRP. The proposed FEQRP achieves much lower number of dropped packets compared to that of the EAGRP; this is because the proposed FEQRP approach balances the energy consumption over all nodes in a fair way and keeps the nodes as alive as possible. Thus, the node failure in the proposed FEQRP is less than that of the EAGRP. The packet dropped was found to be prominent for the EAGRP algorithm as shown in the figure. The proposed FEQRP determines the average energy level of the neighbor nodes. Next, it chooses the forwarding node with high energy level. In this case it can extend the life time of the network in heavy traffic sizes rather than always choosing the node with the maximum weight (progress plus available energy) as in EAGRP. Therefore, it can be observed that the proposed FEQRP achieves low dropped packets. It means that the proposed FEQRP has better performance as the packets increases. 


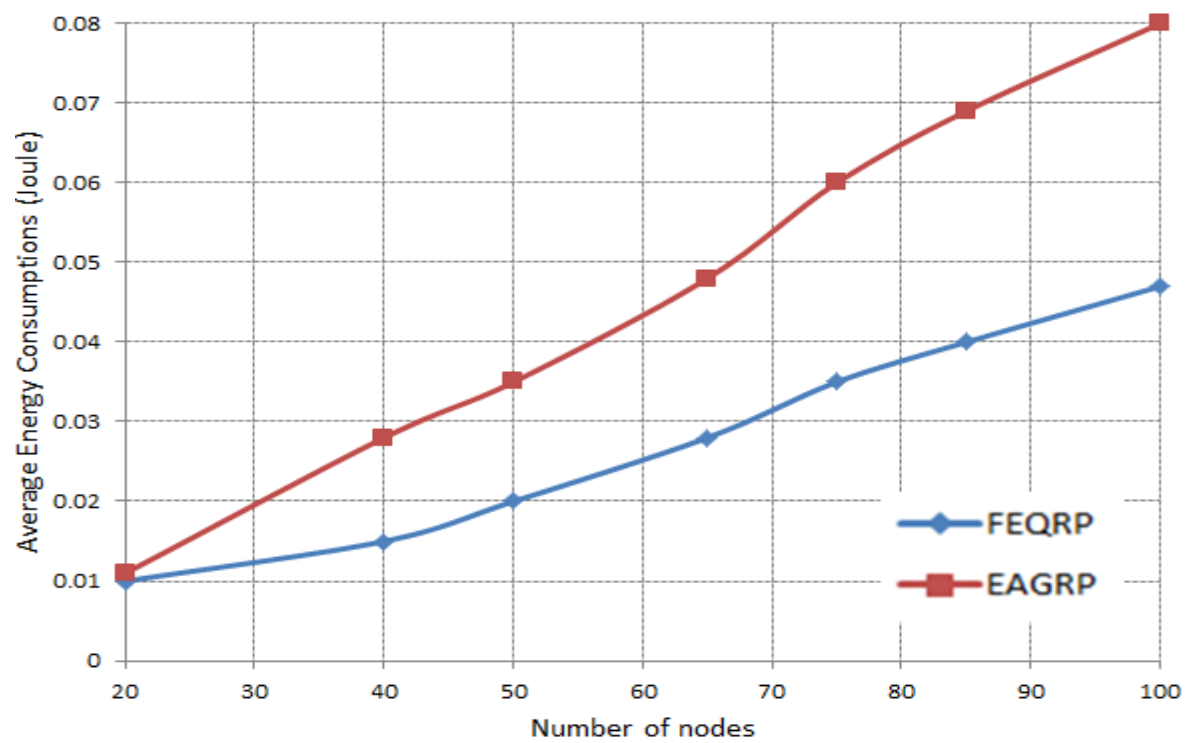

Fig. 2 Average Energy Consumption for both Algorithms in Case of Variable Network Size

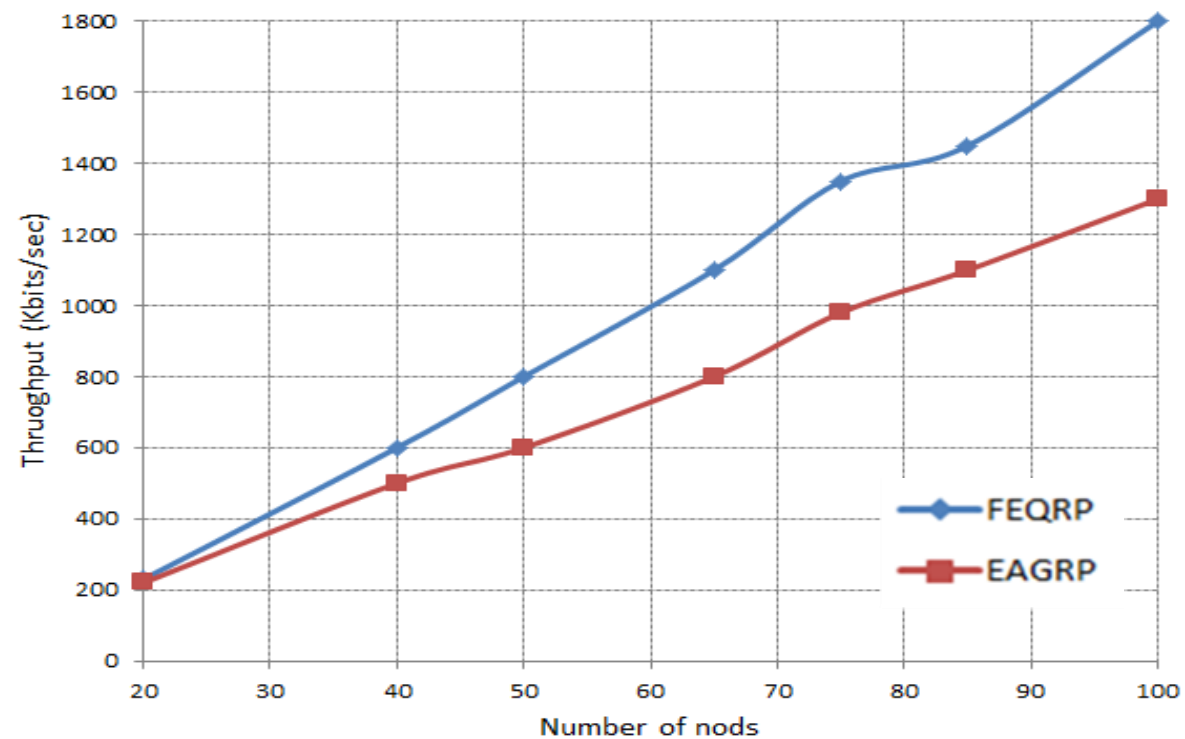

Fig. 3 Throughput for both Algorithms in Case of Variable Network Size

\subsubsection{Average packet delivery ratio}

Fig. 5 shows the average packet delivery ratio of the proposed FEQRP and EAGRP algorithms. It is evident that the proposed FEQRP algorithm provides better data delivery ratio than that of the EAGRP algorithm. The successful packet delivery ratio of the proposed FEQRP is about $93 \%$ on average compared to $80 \%$ for EAGRP. It is mainly affected by the varying size of the network while keeping other parameter constant. It has been observed that the amount of packets delivered is larger with all the network sizes than that of the EAGRP. It means that FEQRP improves the performance much more as the number of source nodes increases. This is because the number of dropped packets of the FEQRP decreased compared to that of the EAGRP. 


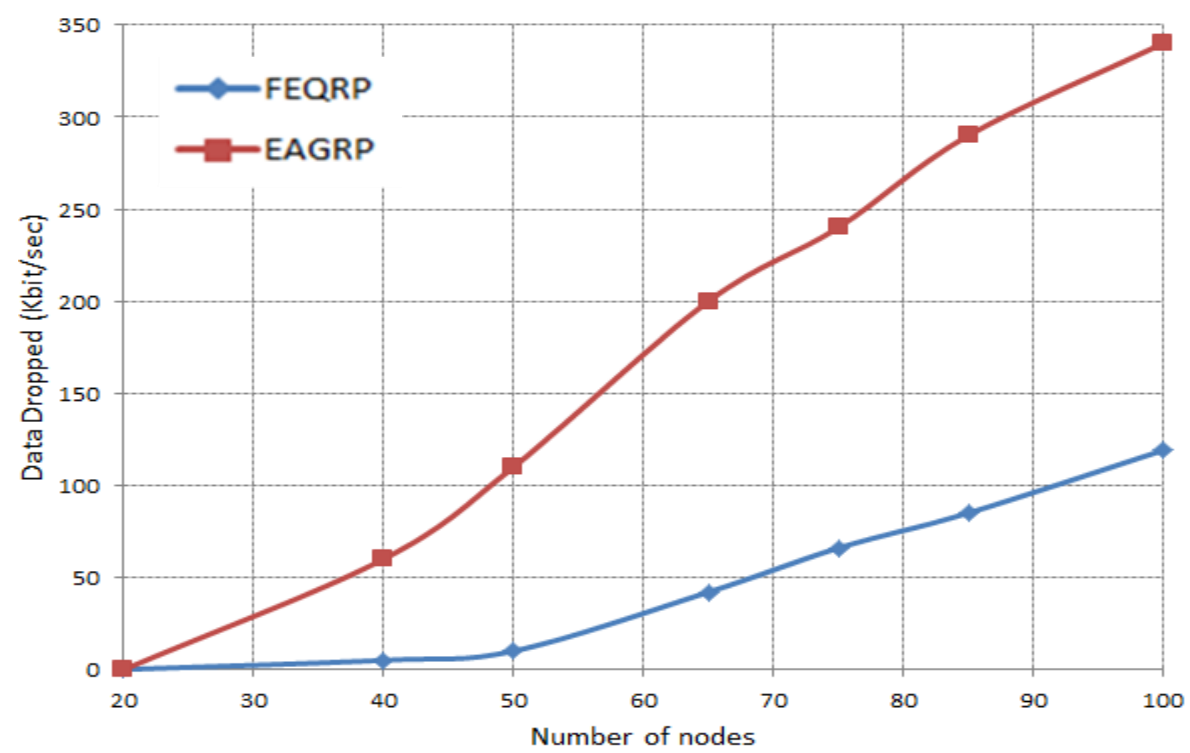

Fig. 4 Data Dropped for both Algorithms in Case of Variable Network Size

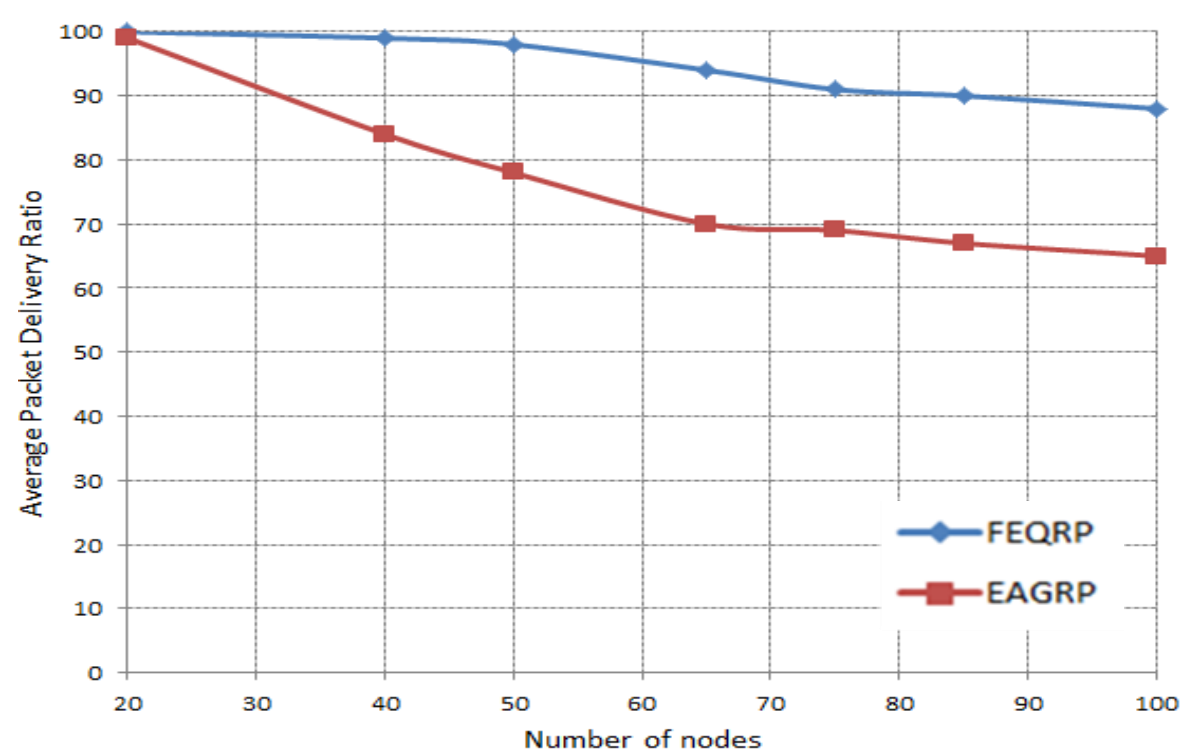

Fig. 5 Average Packet Delivery Ratio for both Algorithms in Case of Variable Network Size

\subsubsection{Average end-to-end delay (sec)}

Fig. 6 presents the delay encountered by the two protocols during the simulation time for all scenarios. It is clear from the figure that the proposed FEQRP algorithm gives lower delay than that of EAGRP algorithm. It always has the smallest delay than that of the EAGRP even when the number of nodes increases. It means that the proposed FEQRP improves the performance much more with the increase of the number of nodes. This is because the EAGRP forwards the data packets to the broken links and thus the average end-to-end delay is affected by the buffering delays. Also the broken links drop the packets which force the source node to retransmit the same data several times and thus increase the delay values.

\subsection{Case 2: Variable Packets Rate with Fixed Network Size}

Here, the five performance parameters will be illustrated for both FEQRP and EAGRP algorithms, where the network size will be fixed to 65 nodes while the packet rate will be variable in the range of $(2,7)$.

\subsubsection{Average Energy Consumption (joule)}

Fig. 7 shows the average energy consumption of the proposed FEQRP and the EAGRP algorithms. It is observed from the figure that the proposed protocol achieves more energy savings than the EAGRP when the packet rate increases. This is because the proposed protocol fairly balances the energy consumption in the network, and thus the FEQRP algorithm saves more energy than that of the EAGRP. As a result, the proposed algorithm will maximize the lifetime of the sensor nodes and thus the network lifetime. 


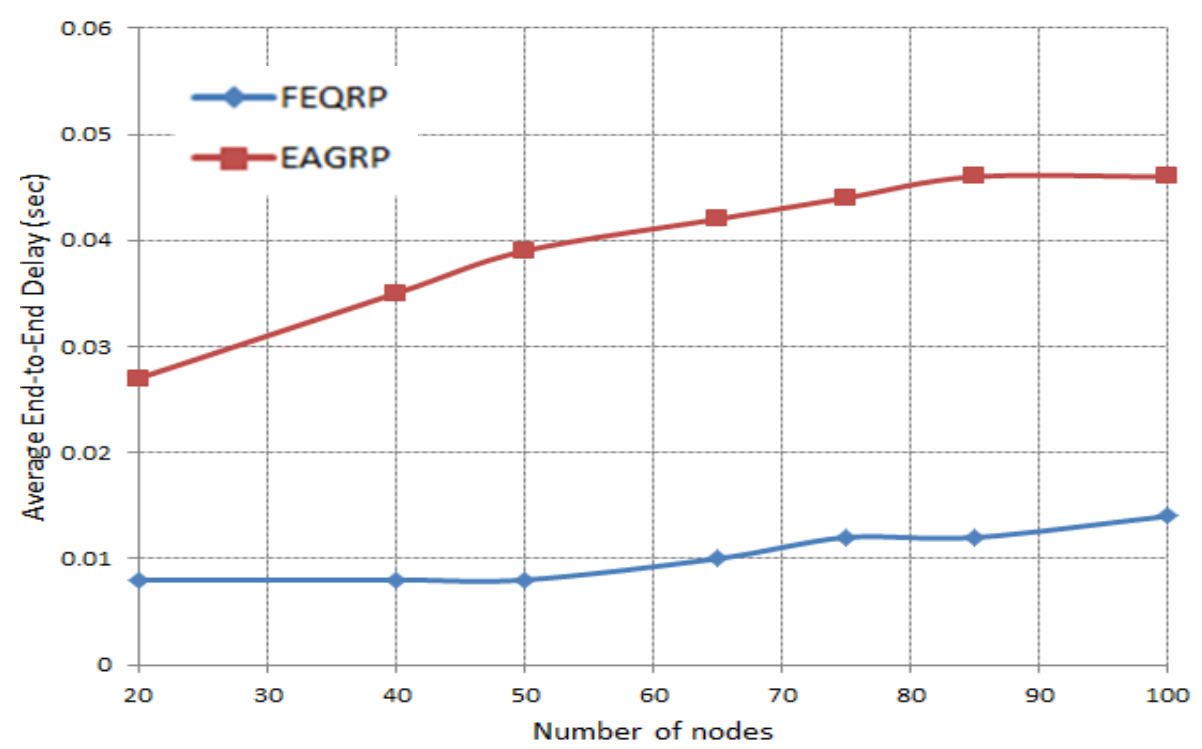

Fig. 6 Average End to End Delay for both Algorithms in Case of Variable Network Size

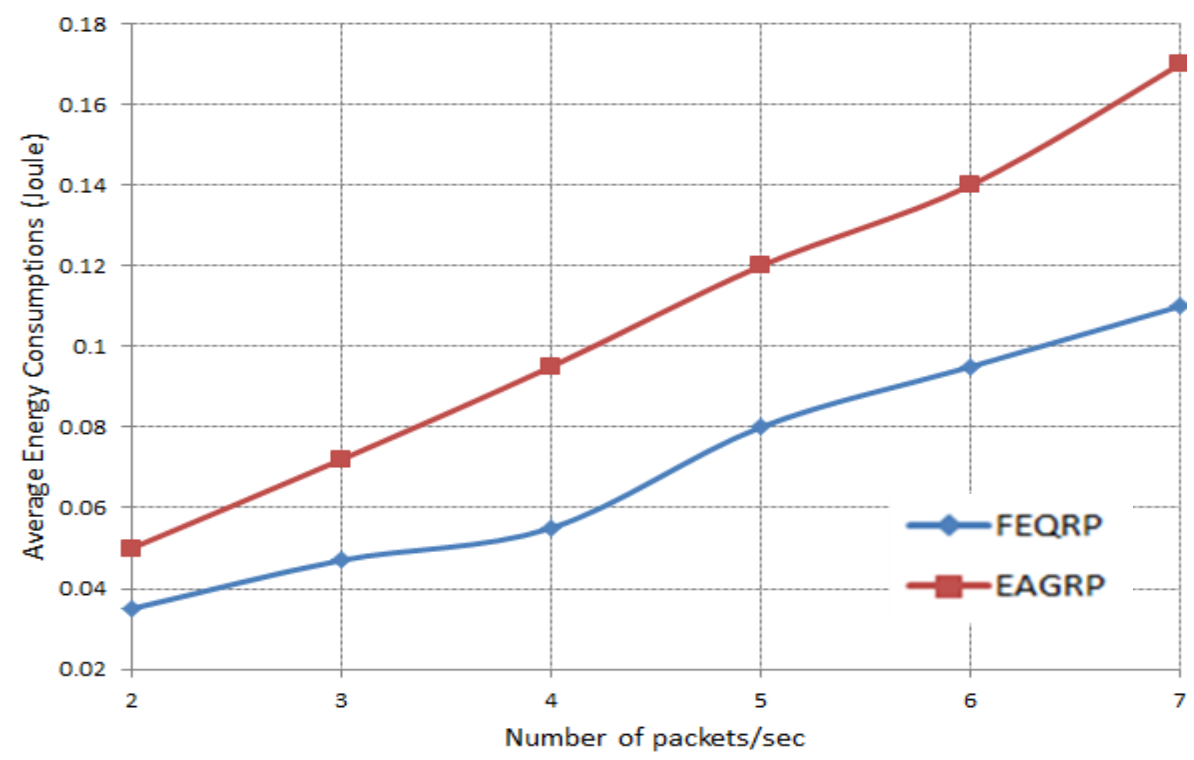

Fig. 7 Average Energy Consumption for both Algorithms in Case of Variable Packet Rates

\subsubsection{Throughput (Kbits/sec)}

Fig. 8 shows the throughput of the proposed FEQRP and the EAGRP algorithms. When the traffic load increases it can be observed that the proposed FEQRP algorithm leads to higher throughput than that of the other. It is shown from the figure that the throughput of the proposed FEQRP is better than that of the EAGRP because the packet loss is more in the EAGRP. This is due to the EAGRP does not take into consideration the energy balancing and therefore it consumes more energy. As a result, the sensor dies early and this leads to network partitioning and consequently to packets loss.

\subsubsection{Data dropped (Kbits/sec)}

The data dropped for the FEQRP and the EAGRP is shown in Fig. 9. From the figure it is observed that the dropped packets are more in the EAGRP algorithm than that of the proposed FEQRP algorithm when the packet rates increase. For example, when the source node transmits 7 packets/sec the size of the dropped packets in the EAGRP is $750 \mathrm{Kbits} / \mathrm{sec}$, while in the proposed FEQRP is $400 \mathrm{Kbits} / \mathrm{sec}$. This is due to the usage of the fuzzy set approach. The fuzzy set approach manages the energy consumption in a fairly way among all the sensor nodes which keeps the packets and relays it to the sink node safely. The lack of energy consumption management in the EAGRP causes the death of some nodes leading to huge number of dropped packets. 


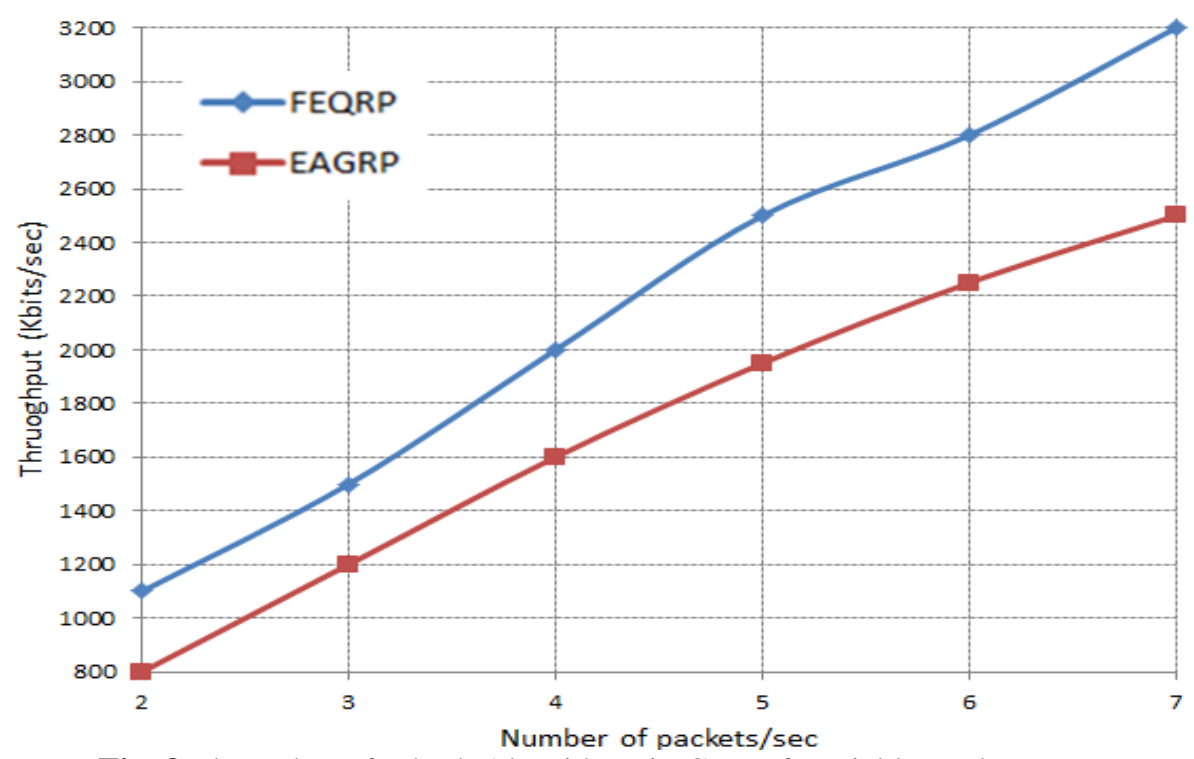

Fig. 8 Throughput for both Algorithms in Case of Variable Packet Rates

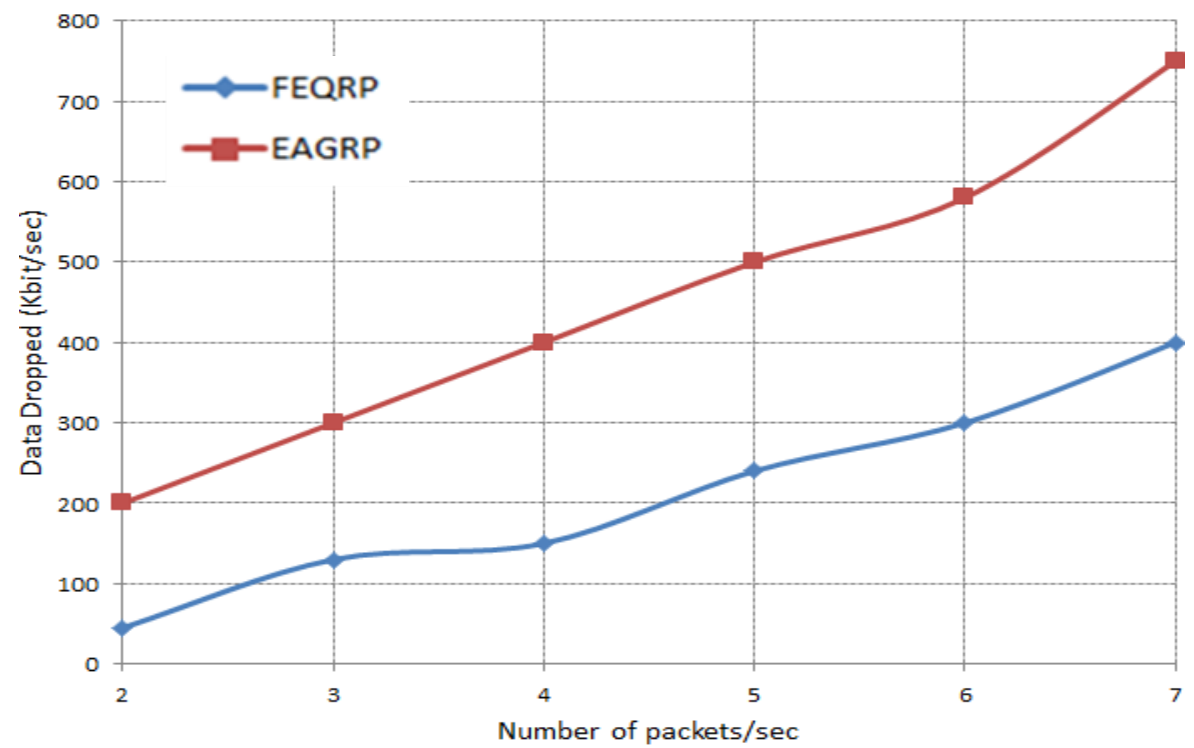

Fig. 9 Data Dropped for both Algorithms in Case of Variable Packet Rates

\subsubsection{Average packet delivery ratio}

In addition to network expansion, increasing the packet rate shows that the FEQRP achieves better performance than that of the EAGRP. In Fig. 10, for example, the successful packet delivery ratio of the FEQRP achieved about $85 \%$ on average compared to $73 \%$ for EAGRP. All this is due to the usage of the fuzzy set approach that selects the next hop according to the high energy level and small distance covered. Thus it prevents the collision of data and keeps the nodes as alive as possible.

\subsubsection{Average end-to-end delay (sec)}

In Fig. 11, the end-to-end delay is plotted against the packets rate. Fig. 11 demonstrates that the average delay is increased directly when the number of packets increases. It has been observed that the value of end to end delay is low for the FEQRP compared to that of the EAGRP. It means that the FEQRP improves the performance much more as the packet rates increase. So the FEQRP algorithm is successful in terms of the average end-to-end delay. This is a result of increasing the amount of dropped packets in the EAGRP which forces the source to retransmit the same data several times, so leads to an increase in the delay values dramatically. 


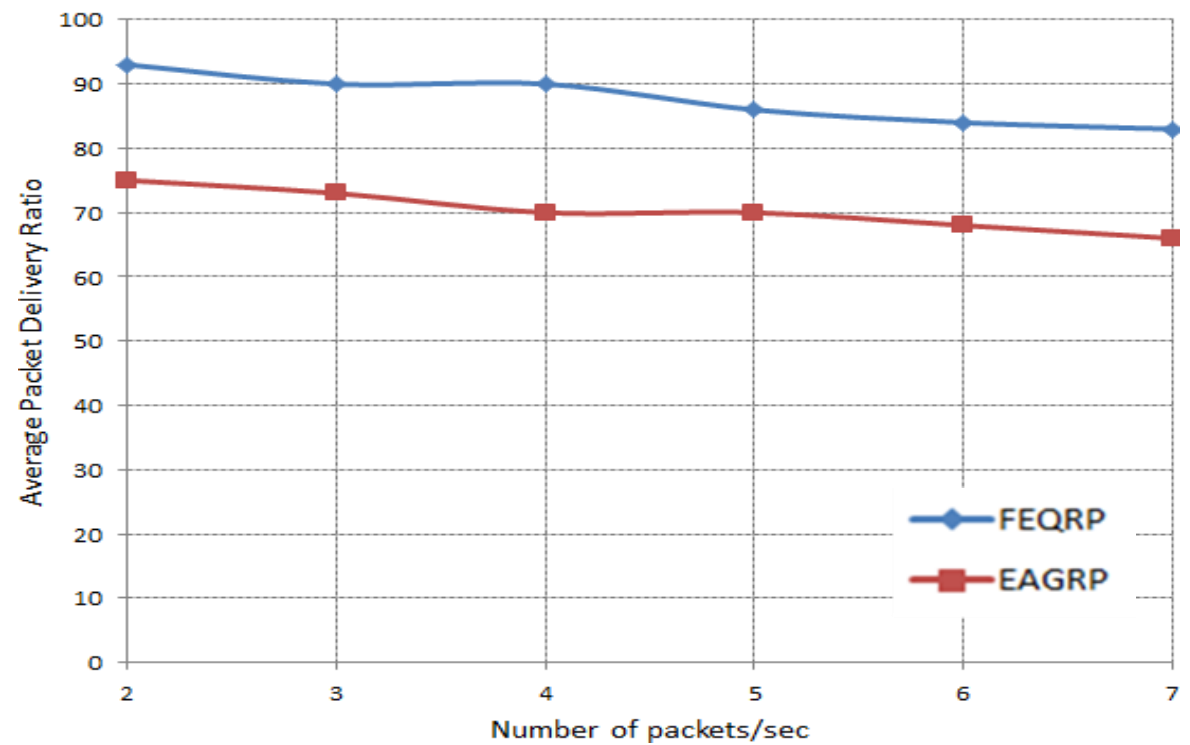

Fig. 10 Average Packet Delivery Ratio for both Algorithms in Case of Variable Packet Rates

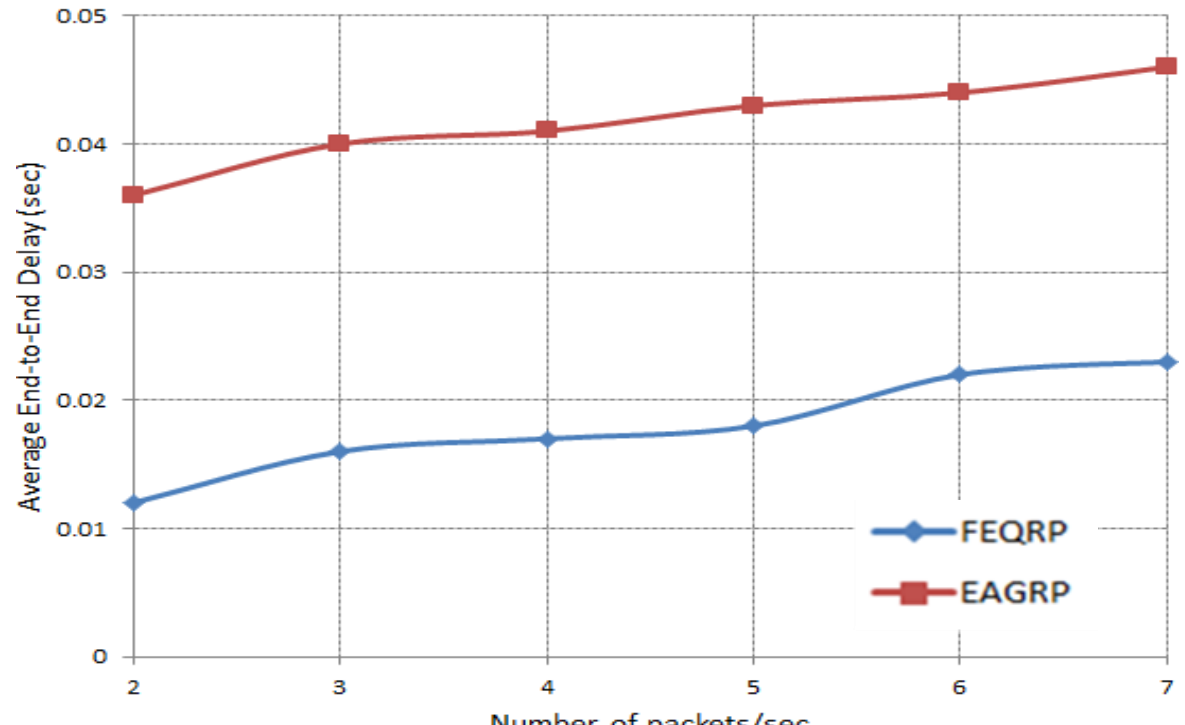

Fig. 11 Average End-to-End Delay for both Algorithms in Case of Variable Packet Rates

\subsection{General Comparison}

Table 2 summarizes the performance of the proposed FEQRP compared to the EAGRP. From the table it is shown that the performance of the proposed FEQRP algorithm is better than the EAGRP algorithm.

Table 2: Percentage Improvement of the Proposed FEQRP Compared to EAGRP

\begin{tabular}{|l|l|l|}
\hline Performance Matric & Case (1 is & Case (2 is \\
\hline Energy consumption & $41.1 \%$ & $34.7 \%$ \\
\hline Throughput & $33.27 \%$ & $27.1 \%$ \\
\hline Dropped packet & $73.06 \%$ & $53.6 \%$ \\
\hline Packet ratio & $17.29 \%$ & $17.33 \%$ \\
\hline End-to-end delay & $75 \%$ & $56.7 \%$ \\
\hline
\end{tabular}

\section{Conclusion}

A new routing algorithm that minimizing energy usage in WSN; named Fuzzy based Energy-efficient and QoS Routing protocol over WSN (FEQRP); was presented in this paper. FEQRP uses the fuzzy set approach to balance the energy consumption and saves energy. FEQRP minimizes the energy consumption and provides QoS routing in WSNs. It is based on location based routing approach.

The proposed FEQRP has been implemented and its performance has been compared with an old algorithm named Energy Aware Geographic Routing Protocol (EAGRP). Two cases were examined, the first 
case uses different number of nodes $(20,40,65,75,85$, and 100) with fixed data rate ( 2 packets/sec), while the second uses different data rates $(2,3,4,5,6$, and 7$)$ with fixed network size (65 nodes). Simulation results have shown that the FEQRP performs competitively against the EAGRP in terms of average energy consumption, throughput, data dropped, average packet delivery ratio and average end to end delay. This is due to the usage of the fuzzy set approach that balance the energy consumption in a fairly way among all the sensor nodes. Therefore it prevents the network partitioning which leads to an increase in the energy consumption, packets loss and delay values.

\section{References}

[1] Kazi C. R., A Survey on Sensor Network, Journal of Convergence Information Technology (JCIT) Journal, vol. 1, no. 1, 2010.

[2] M. Castillo-Effen, D.H. Quintela, R. Jordan, W. Westhoff, and W. Moreno, Wireless sensor networks for flash-flood alerting, Proceedings of the fifth IEEE International Caracas Conference on Devices, Circuits, and systems, Dominican Republic, 2004.

[3] G. Simon, M. Maroti, A. Ledeczi, G. Balogh, B. Kusy, G. Pap, J. Sallai, and K. Frampton, Sensor network-based counter sniper system, Proceedings of the Second International Conference on Embedded Networked Sensor Systems (Sensys), Baltimore, MD, 2004 .

[4] E. Ahvar, A. Pourmoslemi, M. Piran, FEAR: a Fuzzy-based Energy-Aware Routing Protocol for Wireless Sensor Networks, International Journal of Grid Computing \& Applications (IJGCA). vol.2, no.2, June 2011.

[5] L.A.Zadeh, Fuzzy sets, Inform. Control 8, pp. 338-353, 1965.

[6] S. Katayoun, G.Jay, A.Vishal, and J.P. Gregory, Protocols for Self-Organization of a Wireless Sensor Network, UCLA IEEE Personal Communications, vol. 7, Issue 5, pp. 16-27, October 2000.

[7] T. He, et al., SPEED: A stateless protocol for real-time communication in sensor networks, Proceedings of the International Conference on Distributed Computing Systems, Providence, RI, USA, pp. 46-55, 19-22 May. 2003.

[8] B. Karp and H. T. Kung, GPSR: Greedy perimeter stateless routing for wireless networks, Proceedings ACM MobiCom'00, Boston, MA, pp. 243-254, Aug. 2000.

[9] W. Keiss, H. Fuessler and J. Widmer, Hierarchical Location Service for Mobile Ad hoc Networks, ACM SIGMOBILE Mobile Computing and Communications Review, vol. 8, no. 4, pp. 47-58, 2004.

[10] A. A. Elrahim, EAGRP: Energy Aware Geographic Routing Protocol for Wireless Sensor Networks, International Journal of Computer Science and Telecommunications, vol. 5, Issue 11, November 2014.

[11] H.J. Zimmermann, Fuzzy Set Theory and its Applications (third ed. Kluwer Academic Publishers, Boston, MA. 1996). 